\title{
Stability Analysis of Internet Congestion Control Model with Compound TCP Under RED
}

\author{
Dawei Ding ${ }^{1, *}$, Xiaoyun Zhang ${ }^{2}$ and Dong Xue ${ }^{3}$ \\ ${ }^{I}$ Key Lab of Intelligent Computing and Signal Processing, Ministry of Education, Anhui University, Hefei China \\ ${ }^{2}$ School of Electronics and Information Engineering, Anhui University, Hefei, China \\ ${ }^{3}$ Communication Research Center, Harbin Institute of Technology, Harbin, China
}

\begin{abstract}
Many applications require fast data transfer over high speed and long distance networks. Compound TCP (CTCP) is a novel congestion control algorithm for high-speed and long delay networks. This paper develops a discrete time dynamic feedback model of a congestion control system with CTCP under random early detection (RED). We find that periodic doubling bifurcation occurs when varying the RED control parameters or other system parameters. The fixed point of congestion control system and the critical value of parameters are determined by theoretical analysis. Moreover, the result of theoretical analysis is proved and bifurcation and chaotic phenomena are numerically studied by using bifurcation diagrams and Lyapunov Exponent.
\end{abstract}

Keywords: Nonlinear system, Bifurcation, Chaos, Congestion control, CTCP, RED.

\section{INTRODUCTION}

TCP (Transmission Control Protocol) algorithms have been widely used in network for congestion control. TCP can provide reliable data transmission with embedded congestion control algorithm which can effectively adjust the sending rate according to the available bandwidth of the network [1].

However, many applications require fast data transfer over high speed and long distance networks. The initial TCP versions developed for cable networks were not adequate any more. Several improved TCP algorithms have been proposed in recent years. One class of approaches is loss-based which use packet-loss as the only indication of congestion. These algorithms can effectively improve the link utilization while unable to reach RTT fairness. Some typical proposals such as HSTCP [2], STCP [3] and BIC-TCP [4] are of this kind. Another class is delay-based approaches whose congestion decisions are RTT variation based. Some typical proposals include FAST TCP [5], Vegas [6] and BIC [7] which can improve the throughput in high-speed networks may not work well in a mixed environment with both delaybased and loss-based flows.

Compound TCP, as a new congestion control protocol for high-speed and long delay networks, is a combination of both delay-based and loss-based congestion avoidance approach, is called CTCP. Specifically, a scalable delay-based component is added to the standard TCP Reno congestion avoidance algorithm [8]. The delay-based component can

*Address correspondence to these authors at the Key Lab of Intelligent Computing and Signal Processing, Ministry of Education, Anhui University, Hefei 230039, China; E-mail: dwding@ahu.edu.cn efficiently use available bandwidth and react early to congestion by sensing the changes in RTT due to its scalable increasing law $[9,10]$. When the congestion occurs, the delaybased component will gracefully reduce the sending rate and meanwhile the loss-based component keeps the throughput of CTCP lower bounded by TCP Reno. Therefore, CTCP is a promising algorithm to achieve high link utilization while maintaining good RTT fairness and TCP fairness [11].

In this article, we mainly focus on stability region of the system parameter and nonlinear dynamics of a simple network which has only one bottleneck link with CTCP connections and a RED gateway. First, a deterministic nonlinear dynamical model of this congestion control system is introduced. Then, by theoretical analysis, the fixed point and linear stability criterion of this system are derived. Moreover, adopting different RED parameters as bifurcation parameters, the existence of period doubling bifurcation and chaotic behaviors is verified by bifurcation diagram and Lyapunov exponent.

The main contents of this article include the following aspects. Sect. 2 presents the nonlinear discrete-time model used in the analysis. In Sect.3, the fixed point and period doubling bifurcation are analyzed. Sect. 4 presents numerical examples illustrating the nonlinear dynamics in the model. Finally, conclusions are drawn in Sect.5.

\section{DYNAMIC MODEL FOR CTCP UNDER RED}

Consider a simple network where a single bottleneck link is shared by multiple connections. Assume that all connections are long-lived uniform CTCP connections with the same round-trip propagation delay, which is denoted by $\mathrm{d}$ second. The number of these connections is $\mathrm{N}$ and their packet size is $\mathrm{M}$ bit/packer. The capacity of the bottleneck 
link is denoted by C bit/s. Similar to the description in Ref. [12], the congestion control feedback system is defined as follows. At period k, the RED controller at the router provides feedback signal $p_{k}$ (packet drop probability), which is a function of the average queue size $q_{k}$ at period k. Due to the feedback delay of round trip time, the packet drop probability $p_{k}$ at period $\mathrm{k}(\mathrm{k} \geq 1)$ determines the throughput of connections and the queue size $q_{k+1}$ at period $k+1$.

The queue size $q_{k}$ is used to compute the average queue size $\bar{q}_{k}$ according to the exponential averaging rule. Then the average queue size $\bar{q}_{k}$ is used to calculate the packet drop probability at period $\mathrm{k}$. These can be expressed as follows:

$$
\left\{\begin{array}{l}
q_{k+1}=G\left(P_{K}\right) \\
\bar{q}_{k+1}=A\left(\bar{q}_{k}, q_{k+1}\right) \\
p_{k+1}=H\left(\bar{q}_{k+1}\right)
\end{array}\right.
$$

where $A\left(\bar{q}_{k}, q_{k+1}\right)$ is the averaging function:

$$
A\left(\bar{q}_{k}, q_{k+1}\right)=w q_{k+1}+(1-w) \bar{q}
$$

where $w$ is the exponential averaging weight that determines how fast the RED mechanism reacts to a time-varying load.

The RED control function $H\left(\bar{q}_{k+1}\right)$ is given as:

$$
H\left(\bar{q}_{k+1}\right)=\left\{\begin{array}{c}
0 ; \text { if } \bar{q}_{k+1}<q_{\min } \\
1 ; i f \bar{q}_{k+1}>q_{\max } \\
\frac{\bar{q}_{k+1}-q_{\min }}{q_{\max }-q_{\min }} p \text { max } ; \text { otherwise }
\end{array}\right.
$$

where $q_{\min }$ and $q_{\max }$ are the lower and upper threshold values, and $p_{\max }$ is the drop probability when $\bar{q}=q_{\max }$. It has been shown that a CTCP connection provides the following stationary throughput [11]:

$$
T^{C}=\frac{1}{R \alpha^{\frac{1}{2-k}}\left(1-(1-\beta)^{1-k}\right)}\left[\frac{1-(1-\beta)^{\frac{1-k}{2-k}}}{2-k}\right]^{\frac{1-k}{2-k}} \cdot \frac{1}{p^{\frac{1}{2-k}}}
$$

where $R$ is average round trip time:

$$
R=d+\frac{q M}{C}
$$

Parameters of $\alpha, \beta$ and $k$ are tunable to give out desirable stability, smoothness and responsiveness.

Because the aggregate throughput of connections cannot exceed link capacity, the steady-state packet drop probability $P_{u}$ such that the bandwidth capacity constraint is satisfied as:

$$
N T^{C}=\frac{N}{R \alpha^{\frac{1}{2-k}}\left(1-(1-\beta)^{1-k}\right)}\left[\frac{1-(1-\beta)^{\frac{1-k}{2-k}}}{2-k}\right]^{\frac{1-k}{2-k}} \cdot \frac{1}{p^{\frac{1}{2-k}}}=C
$$

The following can then be obtained from Eq. (6):

$$
P_{u}=\left(\frac{N M}{2 C d \alpha^{\frac{1}{2-k}}\left(1-(1-\beta)^{1-k}\right)}\left[\frac{1-(1-\beta)^{\frac{1-k}{2-k}}}{2-k}\right]^{\frac{1-k}{2-k}}\right)^{2-k}
$$

This is the smallest probability that results in a queue size of zero at the next period, i.e., $q_{k+1}=0$, if $p_{k}>p_{u}$. The average queue size $\bar{q}_{u}$, which satisfies $q_{k+1}=0$, if $\bar{q}_{k}>\bar{q}_{u}$, is given by:

$q_{u}=\left\{\begin{array}{c}\frac{p_{u}\left(q_{\max }-q_{\min }\right)}{q_{\text {max }}}+q_{\min } ; i f p_{u} \leq p_{\max } \\ q_{\max } ; \text { otherwise }\end{array}\right.$

Thus, if $p_{k}<p_{u}$, the queue size $q_{k+1}$ at period $\mathrm{k}+1$ can be obtained from the following equation:

$\frac{M N}{R \alpha^{\frac{1}{2-k}}\left(1-(1-\beta)^{1-k}\right)}\left[\frac{1-(1-\beta)^{\frac{1-k}{2-k}}}{2-k}\right]^{\frac{1-k}{2-k}} \cdot \frac{1}{p^{\frac{1}{2-k}}}=C$

From Eq. (9), it can be derived that:

$$
q_{k+1}=\frac{N}{\alpha^{\frac{1}{2-k}}\left(1-(1-\beta)^{1-k}\right) p^{\frac{1}{2-k}}}\left[\frac{1-(1-\beta)^{\frac{1-k}{2-k}}}{2-k}\right]^{\frac{1-k}{2-k}} \cdot\left(-\frac{C d}{M}\right)
$$

From Eq. (10), it can be seen that $q_{k+1}$ is a strictly decreasing function of $p_{k}$. Hence the largest packet drop probability $p_{l}$ such that the queue size $q_{k+1}$ at period $\mathrm{k}+1$ equals the buffer size $\mathrm{B}$ is given by:

$p_{l}=\left(\frac{N}{\alpha^{\frac{1}{2-k}}\left(1-(1-\beta)^{1-k}\right) p^{\frac{1}{2-k}}}\left[\frac{1-(1-\beta)^{\frac{1-k}{2-k}}}{2-k}\right]^{\frac{1-k}{2-k}}\right)^{2-k}$

The corresponding average queue size $\bar{q}_{l}$ is:

$\bar{q}_{l}=\frac{p_{l}\left(q_{\max }-q_{\min }\right)}{q_{\max }}$

From above analysis, it can be derived that:

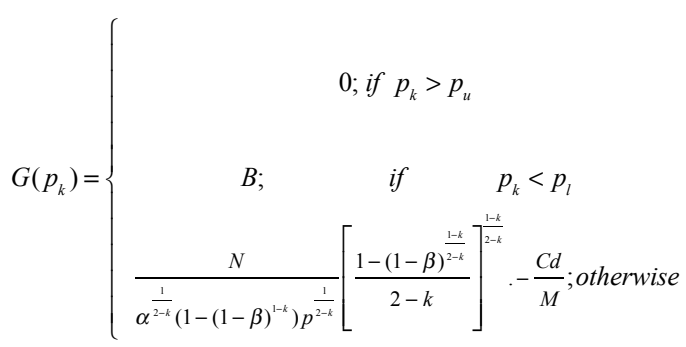

From Eqs.(1)-(3) and Eq.(13), one can obtain the nonlinear one-order discrete-time dynamic model of CTCP under RED: 


$$
\begin{gathered}
\bar{q}_{k+1}=\left\{\begin{array}{cc}
(1-w) \bar{q}_{k}, & \text { if } \bar{q}_{k}<\bar{q}_{l} \\
(1-w) \bar{q}_{k}+w B, & \text { if } \quad \bar{q}_{k}<\bar{q}_{l} \\
(1-w) \bar{q}_{k}+w\left(\frac{\frac{1}{2-k}\left(1-(1-\beta)^{1-k}\right) p^{\frac{1}{2-k}}}{\alpha^{2-1}}\left[\frac{1-(1-\beta)^{\frac{1-k}{2-k}}}{2-k}\right]^{\frac{1-k}{2-k}} \cdot-\frac{C d}{M}\right), \text { otherwise }
\end{array}\right. \\
\text { Where } p_{k}=\frac{p_{\max }\left(\bar{q}_{k}-q_{\min }\right)}{q_{\max }-q_{\min }} .
\end{gathered}
$$

\section{FIXED POINT AND BIFURCATION}

\subsection{Fixed Point of the System} denote

To derive the fixed point of Eq. (14), the authors first

$\bar{q}_{k+1}=g\left(\bar{q}_{k}, \rho\right)$

where $\rho$ is the system parameter such as exponential averaging weight $w$. The fixed point of mapping $g(*)$ is an average queue size $q^{*}$ such that $q^{*}=g\left(q^{*}, \rho\right)$.If the parameters are properly configured, the fixed point should remain between $q_{\max }$ and $q_{\min }$. Solving $q^{*}=g\left(q^{*}, \rho\right)$, one derives that the fixed point of the system is given as a solution to the following polynomial:

$y-e \cdot\left(y-q_{\min }\right)^{\frac{-1}{2-k}}-\frac{C d}{M}=0$

where

$$
e=N\left[\frac{1-(1-\beta)^{\frac{1-k}{2-k}}}{2-k}\right]^{\frac{1-k}{2-k}}\left(\alpha^{\frac{1}{2-k}}\left(1-(1-\beta)^{1-k}\right)\left(\frac{p_{\max }}{q_{\max }-q_{\min }}\right)^{\frac{1}{2-k}}\right)^{-1}
$$

For Eq.(16) is a transcendental equation, we use Matlab to calculate its numerical solution.

\subsection{Bifurcation Analysis}

The linear stability of the fixed point $q^{*}$ can be worked out by the associated eigenvalue $p_{\max }$.

$$
\begin{aligned}
& \left.\frac{\partial_{g}\left(\bar{q}_{k}, \rho\right)}{\partial_{q_{k}}}\right|_{q^{*}}=1-w-\frac{w e}{2-k}\left(q^{*}-q_{\min }\right)^{\frac{k-3}{2-k}} \\
& \quad \text { or } \\
& \left|1-w-\frac{w e}{2-k}\left(q^{*}-q_{\min }\right)^{\frac{k-3}{2-k}}\right|<1
\end{aligned}
$$

Thus, the case is considered in which the linear stability criterion is just violated and a bifurcation occurs, which leads to oscillatory behavior of the system. To illustrate the existence of such bifurcation, one should choose a bifurcation parameter. Several parameters of the system can be chosen as bifurcation parameter, such as the exponential average weight $w$, the number of TCP connections $N$, the lower and upper threshold values $q_{\min }$ and $q_{\max }$, the propagation delay $d$ and $p_{\max }$.

First, one chooses the exponential average weight $w$ as bifurcation parameter. From Eq.(18), it is known that the eigenvalue is a linearly decreasing function of $w$, hence the critical value of $w$ is a value with its eigenvalue given by Eq.(18) is -1 , and one derives that critical value of $w$ is given as a solution to the following polynomial:

$$
\frac{w_{c} e}{2-k}\left(q^{*}-q_{\min }\right)^{\frac{k-3}{2-k}}+w_{c}-2=0
$$

For Eq. (19) is a transcendental equation, we use Matlab to calculate its numerical solution.

When the exponential average weight is increased and exceeds the critical value, the fixed point becomes unstable and a period doubling bifurcation occurs. A period doubling bifurcation has two types: supercritical and subcritical. A supercritical bifurcation leads to a steady oscillatory behavior near the fixed point while a subcritical bifurcation results in divergent oscillations. To determine the nature of the bifurcation, the second and the third derivatives of $g(*)$ are computed:

$\left.\frac{\partial_{g}^{2}\left(\vec{q}_{k}, \rho\right)}{\partial_{\bar{q}_{k}^{2}}}\right|_{q^{*}}=-\frac{w e(k-3)}{(2-k)^{2}}\left(q^{*}-q_{\min }\right)^{\frac{2 k-5}{2-k}}$
$\left.\frac{\partial_{g}^{3}\left(\vec{q}_{k}, \rho\right)}{\partial_{\bar{q}_{k}^{3}}}\right|_{q^{*}}=-\frac{w e(k-3)(2 k-5)}{(2-k)^{3}}\left(q^{*}-q_{\min }\right)^{\frac{3 k-7}{2-k}}$

the quantity:

$$
s=\frac{1}{2}\left(\frac{\partial_{g}^{2}\left(\vec{q}_{k}, \rho\right)}{\partial_{\bar{q}_{k}^{2}}}\right)^{2}+\frac{1}{3}\left(\frac{\partial_{g}^{3}\left(\vec{q}_{k}, \rho\right)}{\partial_{\bar{q}_{k}^{3}}}\right)
$$

determines the nature of the period doubling bifurcation [13]. A positive $s$ indicates that the bifurcation is supercritical and a negative $s$ implies a subcritical bifurcation. For Eq.(15), we can get

$$
s=\frac{w e(k-3)}{(2-k)^{3}}\left(q^{*}-q_{\min }\right)^{\frac{3 k-7}{2-k}}\left(\frac{w e(k-3)}{2(2-k)}\left(q^{*}-q_{\min }\right)^{\frac{k-3}{2-k}} \frac{2 k-5}{3}\right)
$$

It is shown that $s$ may change sign and thus result in a subcritical bifurcation if the parameters are not chosen 


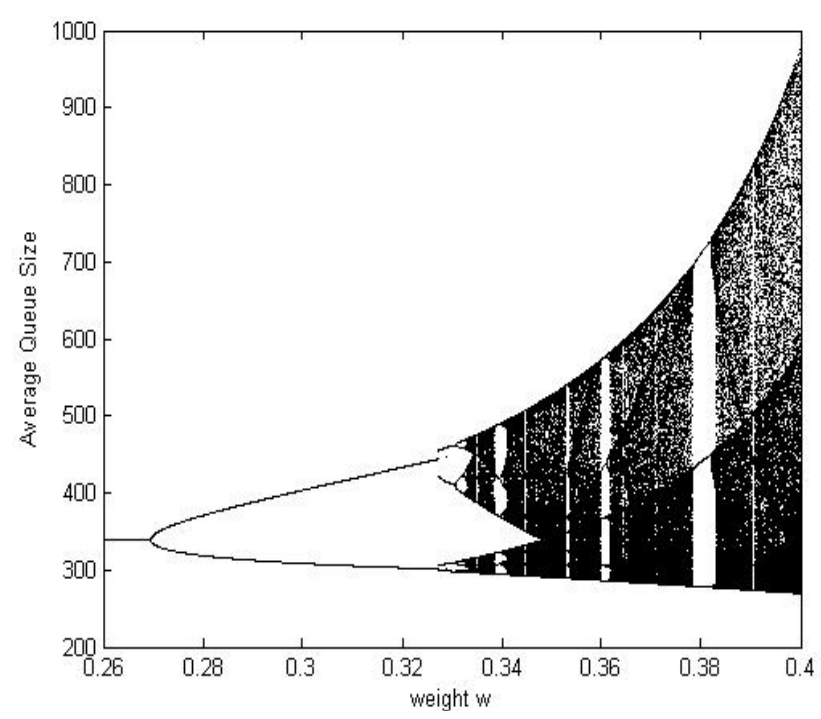

Fig. (1). Bifurcation diagram of average queue size with respect to the exponential averaging weight.

properly. To avoid any unexpected oscillations in router queues, this should be carefully noted when designing a system.

\section{NUMERICAL EXAMPLES}

This section presents numerical examples illustrating the nonlinear dynamics in the model. The stability of the system is numerically studied and the analysis is validated using bifurcation diagrams. A bifurcation diagram shows qualitative changes in the nature or the number of fixed points of a dynamic system with varied parameters.

\subsection{Exponential Averaging Weight}

First, the effect of exponential averaging weight $\mathrm{w}$ is studied. The remaining system parameters are set as follows:

$$
\begin{aligned}
& q_{\max }=750 \text { packets } \\
& q_{\min }=250 \text { packets } \\
& p_{\max }=0.1 \\
& C=15 \mathrm{Mbit} / \mathrm{s} \\
& B=3750 \text { packets } \\
& M=4000 \text { bit } \\
& d=0.1 \mathrm{~s} \\
& N=1
\end{aligned}
$$

and we choose $k=0.75, \alpha=1 / 8$ and $\beta=1 / 2$.

Second, from theoretical analysis in Sect. 3, it is drawn that the fixed point of the system $q^{*}=338.3$, the critical value of $w$ is $w_{c}=0.269$ and $s=0.000245$ at $w_{c}$. The bifurcation diagram is plotted in Fig. (1) with $w$ varying from 0.26 to 0.40 . From Fig. (1), one can see that for $w<w_{c}$, the system is stable and these plots have a fixed point. When $w>w_{c}$, the system loses its stability and a supercritical period doubling bifurcation emerges as $s>0$. This is the initial indication of oscillatory behavior in the system caused by inherent nonlinearity. Increasing $\mathrm{w}$ results in more complex behavior such as chaotic phenomenon. Since a positive Lyapunov exponent indicates the presence of chaos [14], the Lyapunov exponent for the bifurcation scenario is also plotted in Fig. (2). We can see that in the chaos region there exist a large number of periodic orbits.

\subsection{Drop Probability}

In this subsection, how $p_{\max }$ affects system stability and behavior is studied. We set $w=0.28$ and change the value of $p_{\max }$ while other parameters are kept same as those in above subsection. The fixed points of the system with max $p$ varying from 0 to 0.18 are illustrated in Fig. (3), from which it can be seen that similar nonlinear behavior is exhibited in this case as well. However, the plot of fixed point before the first period doubling bifurcation is not a straight line as that in Fig. (1) because the fixed point is varying with changing $p_{\max }$.

\subsection{Lower and Upper Threshold}

In this subsection, we change the lower and upper threshold and investigate its effect on the system. The lower threshold $q_{\min }$ is varied from 150 packet to 450 packet and the upper threshold $q_{\max }=q_{\min }+500$ packets are varied while other parameters are fixed. The bifurcation diagram of 


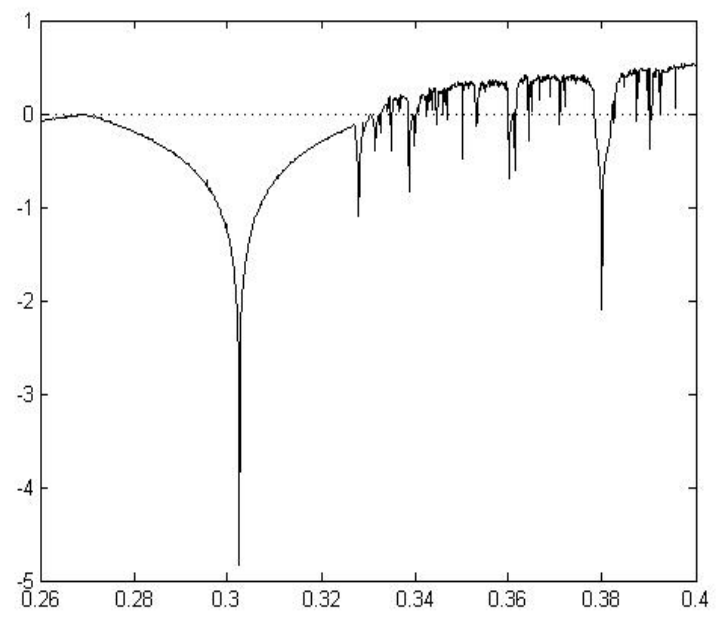

Fig. (2). Lyapunov exponent for average queue size with respect to the exponential averaging weight.

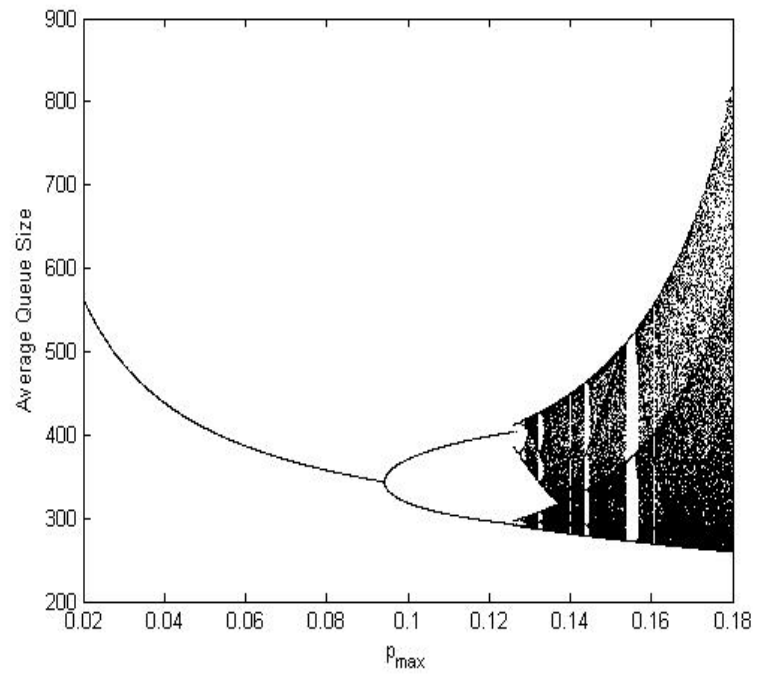

Fig. (3). Bifurcation diagram of average queue size with $p_{\max }$.

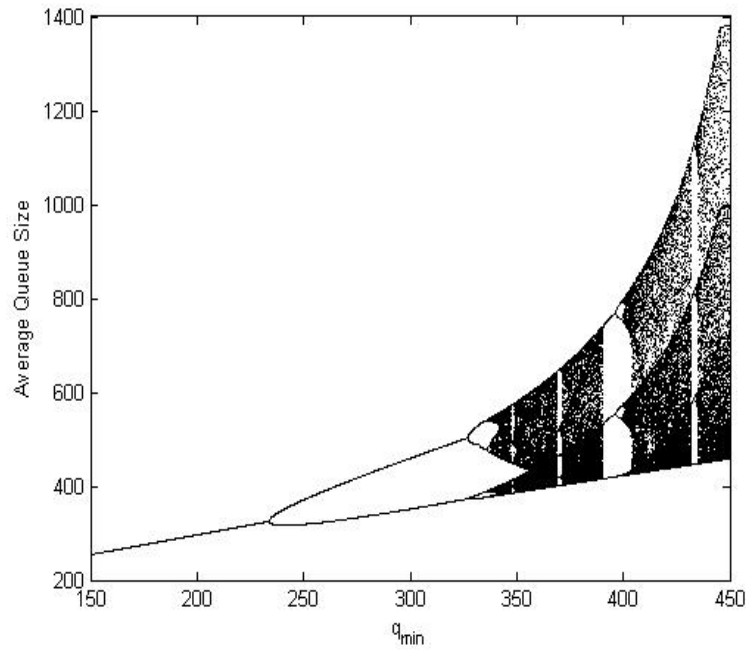

Fig. (4). Bifurcation diagram of average queue size with respect to the lower and upper threshold values. 


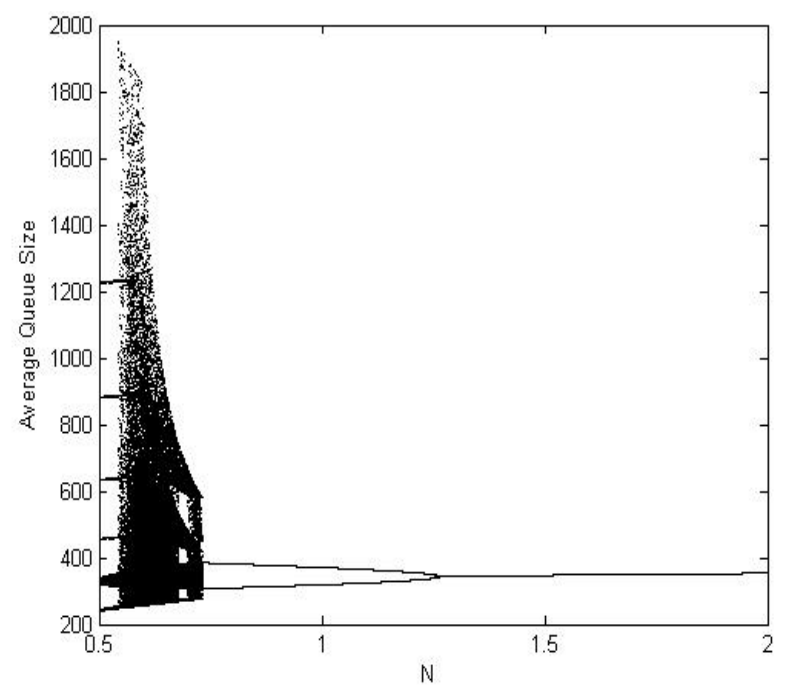

Fig. (5). Bifurcation diagram of average queue size with respect to TCP connections.

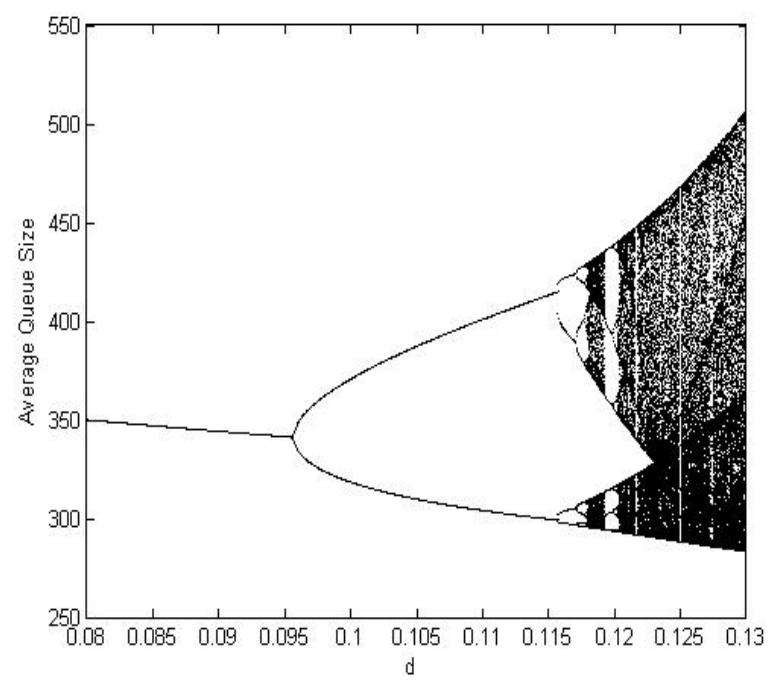

Fig. (6). Bifurcation diagram of average queue size with respect to propagation delay.

average queue size is illustrated in Fig. (4) from which it can be seen that similar nonlinear behavior is exhibited in this case as well. Similarly, the plot of fixed point before the first period doubling bifurcation is not a straight line.

\subsection{Number of Connections}

Unlike the parameter studied above, the number of TCP connections $\mathrm{N}$ and the propagation delay of the networks cannot be controlled by a network manager. Knowing the effect of the number of TCP connections $\mathrm{N}$ and the propagation delay on system stability and behaviors is important for setting the RED control parameters in practice. This subsection studies cover the effect of the number of CTCP connections on the system and in next subsection the effect of the propagation delay of networks is studied.

Here, the number of CTCP connections is varied from 0.5 to 2 . The bifurcation diagram in Fig. (5) shows that the system stabilizes as the number of connections increases. This result agrees with the general result that a larger number of users tend to stabilize the system $[9,10]$.

\subsection{Propagation Delay}

Similarly, the bifurcation diagram is plotted in Fig. (6) with respect to round trip propagation delay. The propagation delay $d$ is varied from 0.08 s to $0.13 \mathrm{~s}$.

\section{CONCLUSION}

In this paper, we mainly deal with the nonlinear dynamics of congestion control system in CTCP connections with RED gateway. First, a one-order discrete time model is developed for this system. Then, the fixed point of the system is determined and the linear stability criterion is obtained. Moreover, some nonlinear behaviors such as chaotic 
phenomena and periodic doubling bifurcation are illustrated by using bifurcation diagrams and Lyapunov exponent. In the future, we may try to employ some control strategies such as hybrid control method [15] for controlling bifurcation and chaos in this model in order to extend the stable parameter range of TCP/RED.

\section{CONFLICT OF INTEREST}

The authors confirm that this article content has no conflict of interest.

\section{ACKNOWLEDGEMENTS}

This paper is supported by the National Natural Science Foundation of China (No: 61201227), NSFC-Guangdong Joint Fund (No: U1201255), the Natural Science Foundation of Anhui (No:1208085MF93) (No:1208085MF93), 211 Innovation Team of Anhui University (No:KJTD007A and No:KJTD001B).

\section{REFERENCES}

[1] M. Allman, V. Paxson, and W. Stevens, "TCP Congestion Control," RFC2581, April 1999.

[2] W. J. Xu, Z. D. Zhou, D.T. Pham, C. Ji, M. Yang, and Q. Liu, "Hybrid congestion control for high-speed networks," Journal of Network and Computer Applications, vol. 34, pp. 1416-1428, July 2011.

[3] R. El Khoury, and E. Altman, "Analysis of scalable TCP congestion control algorithm," Computer Communications, vol. 33, pp. S41-S49, Nov. 2010.

[4] F. Xiao, L. J. Sun, and R. C. Wang, "BIPR: a new TCP variant over satellite networks," The Journal of China Universities of Posts and Telecommunications, vol. 18, pp. 34-39, Sept. 2011.
[5] F. Liu, G. X. Xiong, Z. H. Guan, and H.O. Wang, "Stability analysis and control Hopf bifurcation in a FAST TCP model," In: $32^{\text {nd }}$ Chinese Control Conference (CCC), Xi'an, pp.1076-1080, 2013.

[6] L. S. Xu, K. Harfoush, and I. Rhee, "Binary increase congestion control for fast long-distance networks," In: Proceedings of the $23^{\text {rd }}$ Annual Joint Conference of the IEEE Computer and Communications Societies, Hong Kong, pp. 2514-2524, 2004.

[7] L. S. Brakmo, and L. Peterson, "TCP vegas: end to end congestion avoidance on a global Internet," IEEE Journal on Selected Areas in Communications, vol.13, pp.1465-1480, Oct. 1995.

[8] M. N. I. Khan, R. Ahmed, and M. Aziz, "A survey of TCP Reno, new Reno and sack over mobile ad-hoc network," arXiv preprint arXiv: 1205.3125, International Journal of Distributed and Parallel Systems (IJDPS), vol. 3, Feb. 2012 [Online] Available From: www. arxiv.org

[9] S. Floyd, and K. Fall, "Promoting the use of end-to-end congestion control in the internet," IEEE/ACM Transition on Networking, vol.7, pp.458-472, 1999

[10] J. Mo, R.J. La, V. J. Anantharam, and J. Warrand, "Analysis and comparison of TCP Reno and Vegas," In: $18^{\text {th }}$ Annual Joint Conference of the IEEE Computer and Communications Societies, New York, pp. 1556-1563, 1999.

[11] K. Tan, J.M. Song, Q. Zhang, and M. Sridharan, "A compound TCP approach for high-speed and long distance network," In: 25th IEEE International Conference on Computer Communications, Barcelona, pp. 1-12, 2006.

[12] D. W. Ding, J. Zhu, X. S. Luo, and L. S. Huang, "Nonlinear dynamics in internet congestion control model with TCP Westwood under RED," The Journal of China Universities of Posts and Telecommunications, vol. 16, pp. 53-58, Aug. 2009.

[13] S.J. Guckenheimer, and J.P. Holmes, "Nonlinear oscillations, dynamical systems, and bifurcations of vector fields," Springer-Verlag New York, pp. 221-222, 1983.

[14] Z. Hu,, C. Zhang, G. Luo, Z. Teng, and C. Jia, "Characterizing cross-scale chaotic behaviors of the runoff time series in an inland river of Central Asia," Quaternary International, vol. 311, pp. 132139, Oct. 2013

[15] D.W. Ding, J. Zhu, and X.S. Luo, "Hybrid control of bifurcation and chaos in stroboscopic model of Internet congestion control system," Chinese Physics B, vol. 17, pp. 105-110, Jan. 2008.

Received: May 16, 2015

(C) Ding et al.; Licensee Bentham Open.

This is an open access article licensed under the terms of the (https://creativecommons.org/licenses/by/4.0/legalcode), which permits unrestricted, non-commercial use, distribution and reproduction in any medium, provided the work is properly cited. 